7. It is most important to follow plasma calcium and phosphorus levels about twice weekly post-operatively. The calcium gives the best measure of the success of operation and of the subsequent recovery of remaining normal parathyroid tissue. The phosphorus level usually remains low for some time after and does not help much in management. A rise to abnormally high levels suggests temporary hypoparathyroidism (unless it can be adequately explained as due to worse renal function) and means that therapy may be required to correct hypocalcaemia even if there is no bone disease present.

8. Temporary post-operative correction of calcium and phosphorus levels with subsequent return to preoperative levels indicates that another parathyroid tumour has been left behind and is situated near the site of operation. No change in calcium and phosphorus levels means that a tumour remains far from the site of operation, or else that a misdiagnosis has been made.
9. Alkaline phosphatase levels should be followed every one or two weeks when they were raised preoperatively. A fall to normal means that the bone disease is nearly healed and there is no likelihood of a recurrence of tetany. If vitamin $\mathrm{D}$ is being given this can usually be stopped. In some cases many months may elapse before this occurs.

10. Rarely, signs resembling tetany persist for a short time post-operatively when plasma calcium levels are nearly normal. This may be due to $\mathrm{Mg}$ lack and will then respond to intravenous $\mathrm{MgSO}_{4}$. One should always bear $\mathrm{Mg}$-lack in mind if a patient's mental and neuromuscular symptoms do not immediately respond to treatment of hypocalcaemia.

We note with interest that Jackson and Doncaster (1962) also recommend vitamin $\mathrm{D}$ as treatment for post-operative hypocalcaemia.

[The conclusion of this lecture, together with a list of references, will appear in our next issue.]

\title{
EPISODIC, POSTURAL, AND LINKED ANGINA
}

BY

\section{K. SHIRLEY SMITH, M.D., F.R.C.P.}

CORNELIO PAPP, M.D.

\section{From the Cardiac Departments of Charing Cross and London Chest Hospitals}

The realization that pains in the chest, independent of effort, sometimes lancinating and sometimes long-lasting and often situated below the left breast, were not of cardiac origin was part of the new look that cardiology assumed forty years ago. It did, however, promote the notion that by contrast anginal pain was of two broad kinds : a repetitive paroxysmal type related to effort or pains unrelated to effort that were recurrent and more and more persistent as a prelude to infarction of the heart. As to diagnosis, this did not generally remain long in doubt in either instance. Further, it has generally been accepted that if a patient has anginal pain that is independent of effort, then a sufficient grade of effort, notably locomotion, will surely provoke it.

We have been interested in studying and defining a third variety of angina which is clinically separate from effort angina and also from the worsening anginas (with or without effort) that terminate in overt cardiac infarction. We now bring together as a group those syndromes in which pain proved to emanate from the heart occurs sporadically, independently of exertion, often without any evident provocation and sometimes connected with posture. In this group of disorders the recurrent pains may appear at long intervals and without deterioration of effort tolerance over years. Sometimes attacks are related to cold or to emotional causes; at times they develop only in recumbency, and at times they are closely associated with a disorder that is not related to the coronary system or the heart at all, such as gallbladder disease, cervical spondylitis, and gastrooesophageal disorders.

\section{Criteria, Definitions, and Aims}

In making this study we have set ourselves certain conditions, definitions, and requirements. First, we do not seek to question the well-established neurogenic and non-cardiac basis of the "left submammary" pains. Secondly, we are not dealing with any syndromes that cannot be shown at once or in time to have a basis in coronary artery disease. Such proof may appear in straight cardiography, in or out of the attack, or by cardiographic effort test, or by the ultimate development of cardiac infarction in circumstances that indict the earlier pains as anginal. Thirdly, we exclude from this study general myocardial anoxia due to extracardiac causes and anginal syndromes caused by non-coronary cardiac disease, such as aortic stenosis.

In considering disorders of the gullet, stomach, biliary tract, or the nervous system in connexion with angina, we emphasize that we are not concerned with differential diagnosis. Thus we are not interested to exonerate the heart in any particular disorder but to study patients who admittedly have a coronary disorder but who have, in addition, one of these other syndromes and to demonstrate how these maladies interact. Naturally we have in mind the well-established criteria of noninfarction angina-namely, that the pain is repetitive and of a pattern, even if at long intervals; that it is measured in minutes (not seconds); that it is often generally constricting rather than locally cramping; that its quality is frequently burning but never lancinating; that it has a distracting and disturbing quality, even if it is not a severe pain; and that it vanishes completely when the paroxysm is over, never being associated either in or after the attack with local tenderness.

\section{Classification}

For the purposes of this study we propose the following classification of angina independent of effort : (1) episodic angina (which may be nocturnal and then falls in group $2(a)$. (2) Postural angina: (a) nocturnal or decubitus, and (b) stooping. (3) Linked angina associated with (a) gastro-oesophageal and duodenal disorders and diseases, (b) gall-bladder disease, (c) cervical spondylitis, and (d) left submammary pain. (4) Status anginosus. 
This last is included in the classification for completeness, "yor it is more often than not associated with angina of effort. It is the most severe form of decubital angina, frequently terminating in cardiac infarction, though this may be delayed by months or years (Papp and Shirley Smith, 1960), during which time there is often recurrent angina independent of effort.

\section{Episodic Angina}

This syndrome is one in which pains having the characters of angina of effort occur at longer or shorter intervals. Each attack is similar to the others although sometimes the first bout is more severe than succeeding ones. Whether this is so or not, the disorder may recur over a course of as much as ten or even fifteen years, the patient still having a good effort tolerance, not limited by pain. There may or may not be some recognized circumstance that seems to provoke attacks. The pain is likely to be as severe as that of effort angina, and it is more prolonged; the patient with effort angina can bring the bout to an end by halting, but in episodic angina he has no parallel remedy, although glyceryl trinitrate may be of value. Like effort angina, it will tend to radiate to the back, to the shoulders and elbows, and to the jaws. The resting cardiogram may be normal or abnormal. If it is normal, repetition after an exercise test may show the changes typical of myocardial ischaemia. Even if the test is negative a coronary basis is not excluded: as the pain is never evoked by day-today effort, why should exercise produce patterns of ischaemia? But clinical and instrumental methods alike may fail to clinch the diagnosis, and then only the turn of events may show, as when one of the habitual attacks is prolonged into a proved cardiac infarction. The following examples of sporadic angina may be quoted.

Case 1.-A man of 48 complained that for two months he had had attacks of tightness across the chest, associated with heaviness and numbness in the arms. These bouts would occur at times of agitation or worry or if he had taken spirits to excess the previous night. He denied that effort ever provoked the symptoms. The first bout had been the most severe and had lasted 20 minutes. Thereafter the attacks might occur several times in a day or he might be free for three or four days. On clinical examination the heart was normal. The blood-pressure was 170/110, and there was arteriosclerosis and albuminuria. The cardiogram showed suppression of $T$ in lead I and after an exercise test (which did not evoke the symptoms) there were patterns in leads II, $V_{6}$, and $V_{5}$ denoting mild ischaemia (Fig. 1). Sporadic anginal pains persisted in the same manner and effort angina was never experienced. One evening, two and a half years after the onset, he had a feeling of impending

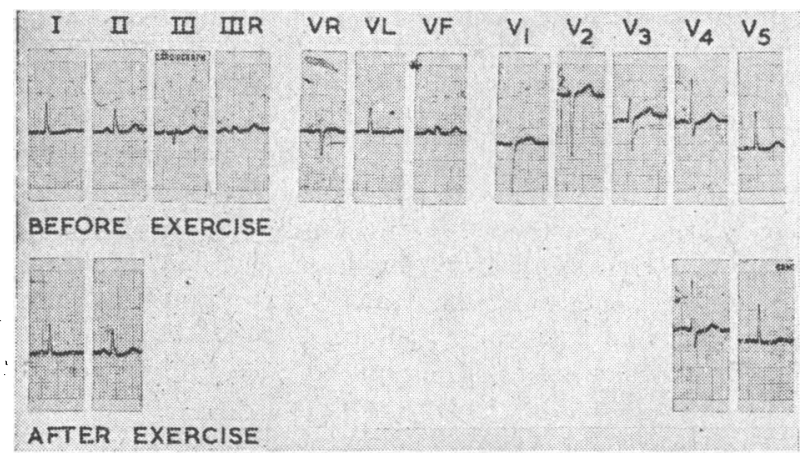

Fig. 1.-Case 1. Cardiographic effort test is positive but shows only minor changes. Sudden death from cardiac infarction proved by necropsy. disaster and was found dead the next morning. The coroner's necropsy disclosed acute coronary occlusion as the cause of death.

Case 2.-A man of 36 had experienced at intervals for three and a half years attacks of thoracic pain starting with a cramp at the left breast and spreading up to the left side of the neck and face and jaw and to the left axilla and arm. Each attack was of the same pattern, usually beginning when the patient was lying or sitting. He found that standing up relieved him. The effort tolerance had always been good and he played tennis with enjoyment, no attack of pain ever having started during physical exertion. In spite of his age, it could not be doubted that this was episodic angina; at first it had been fairly frequent, but the intervals had gradually extended to about three months. The weight was correct and the blood-pressure 125/80. No abnormal signs were found in the heart. The cardiogram was normal and an exercise test produced changes insufficient to class as a positive result. This series of attacks culminated in one that was at first identical with the others but which was longlasting and accompanied by sweating ; in this, the cardiogram showed patterns of acute antero-septal infarction of the heart.

Case 3.-A pathologist aged 55 had for four years been experiencing transient tight sensations across the chest. Although he led an active life, walking and gardening, these sensations, which were sometimes painful, had never occurred during exertion. Eight days before he was first seen this same symptom had occurred while he was in his office; it was the most severe bout he had had and lasted about five minutes. He then felt well again, but thereafter on most days the pain recurred, never during exertion, never long-lasting, and never with any manifest provoking cause. The clinical examination was negative except for mild arteriosclerosis; the blood-pressure was 130/85. The heart was not enlarged. In the cardiogram there were minor abnormalities accentuated by an exercise test on stairs (which caused no pain or tightness) (Fig. 2). The patient was confined to bed for a fortnight, and since then, over a period of five years, there has been no recurrence of the chest symptoms.

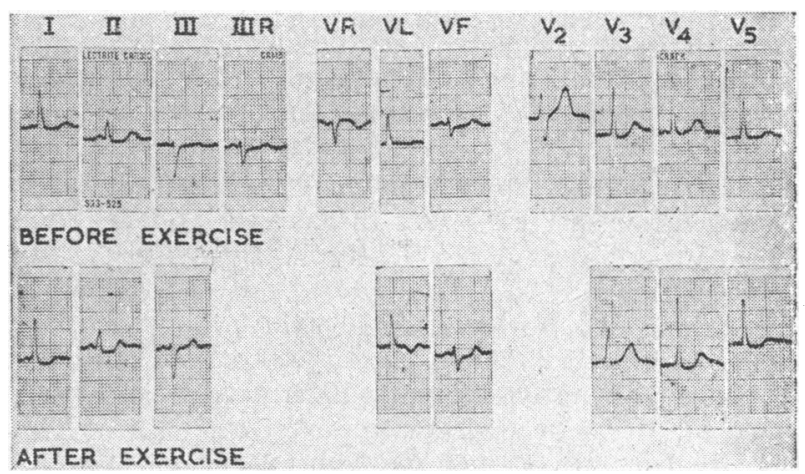
Fio. 2.- Case 3. Cardiographic effort test is positive although
angina was never provoked by effort. Subseguent recovery with freedom from angina. The record incidentally shows the WolffParkinson-White syndrome which does not influence the interpretation.

The foregoing case reports show that episodic angina may be succeeded by cardiac infarction. Conversely, the attacks may become less frequent and less severe, ultimately vanishing. Syndromes of this kind may be obviously associated with exposure to cold or other atmospheric conditions, to sudden agitation or prolonged fatigue or worry, to unaccustomed quantities of alcohol, or to excessive use of tobacco.

\section{Postural Angina}

Postural angina occurs in two forms: (a) nocturnal or decubitus angina, and $(b)$ in relation to certain postures, 
notably stooping. We are considering here only those patients with postural angina who have not experienced effort angina.

\section{Nocturnal Angina}

Nocturnal angina is a designation preferred to angina of decubitus because, in general, it is lying down and sleeping that is the prelude to a seizure, although there are exceptions.

A classical example of nocturnal angina has been described by Gallavardin (1925). A man of 50 suffered from essential hypertension of severe grade. In the course of eight years he had 400-500 attacks of angina, often prolonged and extremely painful. These occurred only in the night and he never had the least thoracic pain or distress by day while walking. He died suddenly in his chair after a meal. Why these circumstanees should evoke cardiac pain is not clearly understood; it is thought that the increased venous return during the night due to reabsorption of fluid deposited in the tissue spaces during the day is responsible for the deterioration of the coronary circulation (Reeves and Harrison, 1956). The cause may thus be similar to that of cardiac asthma; in fact, the two conditions are sometimes associated and diuretic treatment may improve nocturnal angina. It has been stressed that nocturnal angina is particularly frequent in coronary ostial stenosis (Bedford, 1958), and this is perhaps explained by the reduced cardiac output during sleep. Dreaming (whether recalled after waking or not) might be a reasonable explanation and might place the patient on the same footing as one suffering from sporadic angina evoked by mental stress or emotion. The following are characteristic examples of nocturnal angina.

Case 4.-A business man aged 63 had an antero-septal infarct in 1952 and a postero-lateral infarct in 1955 ; he had been on anticoagulant treatment ever since. For the last two years he had been liable to wake at night with acute sternal pain, spreading to the throat; he had to sit up and take glyceryl trinitrate and the pain then subsided within ninutes. Occasionally he might have two or three attacks during the night. Weeks, sometimes months, might elapse between these nocturnal attacks. There had never been associated angina of effort, though the life he lived involved stairs and walking. The cardiogram showed the relics of past infarctions and had not altered for years. In October, 1959, the seizures became more frequent and prolonged; the cardiogram showed the patterns of subendocardial ischaemia, so often seen in status anginosus. He died two months later, a few hours after an acute posterior infarction.

Case 5.-A housewife aged 69, who lived in fear of coronary disease after the sudden death of her husband from cardiac infarction, had yearly cardiograms recorded because of left mammary pain. These and cardiographic effort tests were normal up to December, 1956. In August, 1957, she awoke one night with severe chest pain which was vice-like and which radiated to both arms, lasting 5 to 10 minutes. Successive attacks were easily suppressed by taking glyceryl trinitrate, and she improved with sedation and pentaerythritol tetranitrate. These nocturnal attacks subsequently recurred at intervals of two to three weeks. She continued to do her own shopping, having to climb a bill to reach her home, and yet she has never had any chest pain on effort. It was doubted whether the attacks were anginal, but in December, 1958, an electrocardiogram recorded on the morning after a nocturnal attack showed clear signs of cardiac ischaemia.

Case 6.-A woman secretary aged 52, with a bad family history of coronary disease, experienced in 1953 a squeezing chest pain when running after a bus; it persisted for some time as she sat in the bus and it made her feel ill. On following nights she woke up with similar pains. During the next five years she had no discomfort and was able to enjoy walkıng in the mountains. From October 10 to 24, 1958, she was awakened on six nights by a tight pain in the mid-sternal region which she recognized as similar to the pain she had had five years previously. Glyceryl trinitrate suppressed the pain within minutes. She had a normal blood-pressure but no clinical or radiological signs. The cardiogram showed prolonged Q-T interval, flat-topped $T$ waves in $V_{2}$ and $V_{3}$, and absent $T$ waves in $V_{5}$ and $V_{6}$. She is still a keen walker and never experiences chest pain on effort.

\section{Angina of Stooping}

Stooping or bending during pregnancy may causo faintness through diminished venous return, the inferior vena cava being compressed by the pregnant uterus. In a patient with deficient coronary circulation, even small variations of cardiac output caused through similar mechanisms may be responsible for angina. That stooping causes changes in circulatory dynamics is shown by the behaviour of blood-pressure. In general,

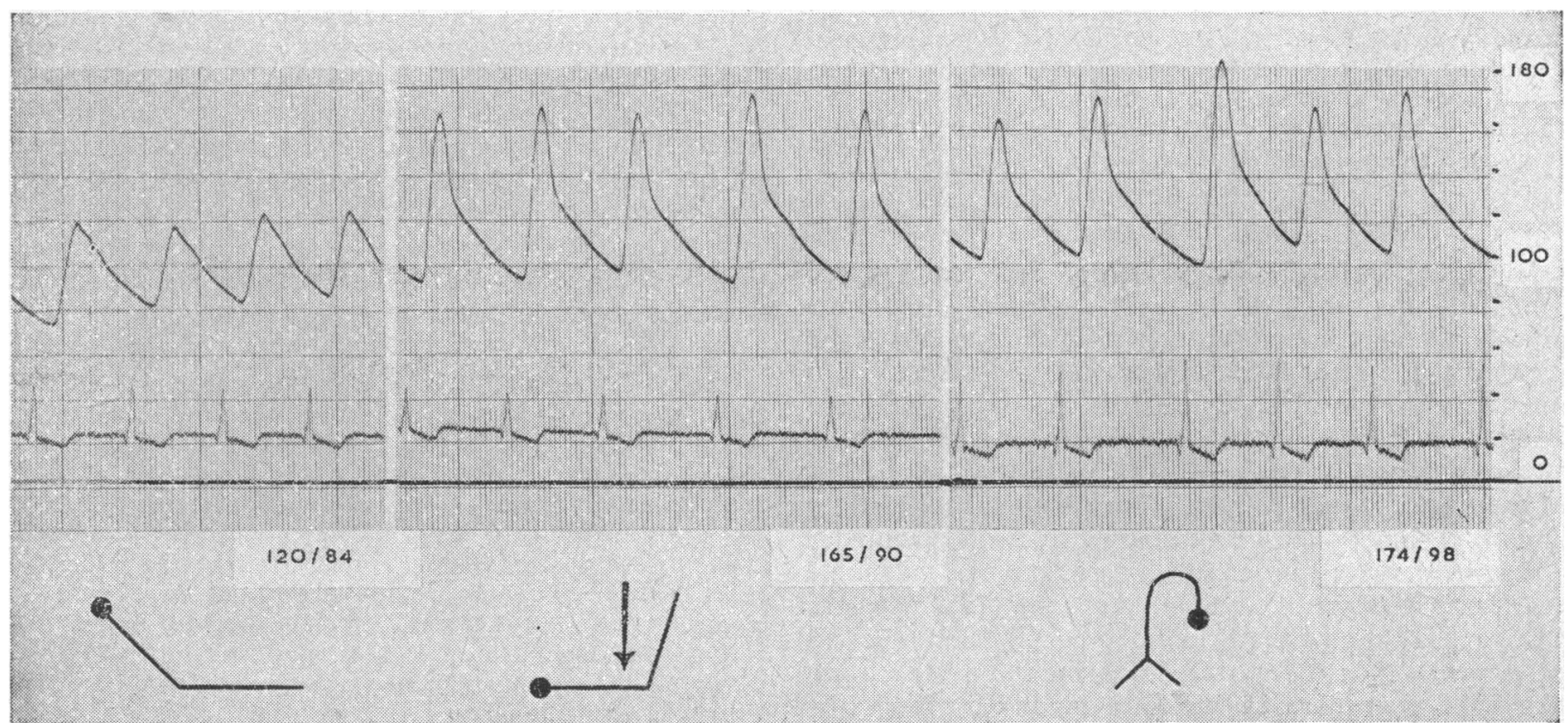

Fro. 3.-Brachial artery tracing and electrocardiogram in a patient with angina of stooping, showing rises of systolic and diastolic blood-pressure during pressure on abdomen with leg-raising and also on stooping. 
the diastolic and systolic pressures rise while the pulsepressure tends to increase, but less consistently. The variations apply equally to patients with postural angina and to healthy controls. The pulse rate during stooping rises more consistently in control subjects than in those with postural angina, in whom it is little, if at all, increased (Shirley Smith and J. W. Mickerson, unpublished). The kind of blood-pressure responses observed when the patient lies, stands, stoops, squats, stands, and lies again are illustrated by the brachial artery tracing shown in Fig. 3. A man with myocardial ischaemia is placed in succession (1) into semi-reclining position, (2) in the thigh-flexed position with abdominal compression, and (3) in the stooping posture. The systolic pressure rises, and later the diastolic.

Case 7.-A man aged 60 had posterior cardiac infarction in 1952 and 1954. In 1955 his main complaint was of tight chest pain on bending to tie shoelaces or when stooping at gardening. On testing, the stooping posture caused a rise of $32 \mathrm{~mm} . \mathrm{Hg}$ in the systolic and $20 \mathrm{~mm} . \mathrm{Hg}$ in the diastolic pressure (Fig. $4 \mathrm{~A}$ ), with a pulse rate increasing from 90 to 110 .
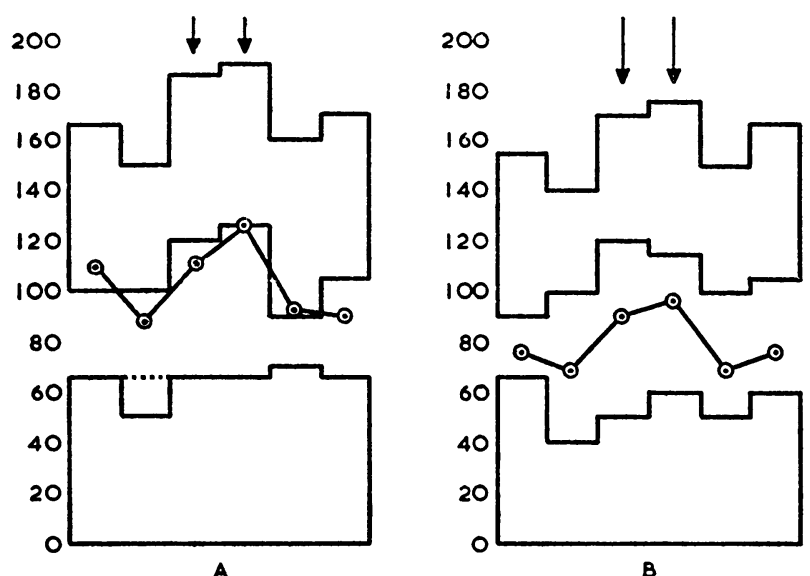

A

Fig. 4.-Cases 7 (A) and 8 (B). Postural angina in men aged 60 and 49 years respectively with coronary disease, showing increase of systolic and diastolic pressure on stooping and squatting. Arrows indicate duration of pain. The six phases

are: lying, standing, stooping, squatting, standing, lying.

Case 8.-A waiter aged 49 had a posterior cardiac infarction. Soon after his recovery he was pulled up by sternal pain after walking 100 yards. Thereafter his main complaint was of sternal pain after meals and on stooping to lift trays or to tie his shoes. In Fig. $4 \mathrm{~B}$ are shown the changes in blood-pressure with change of posture, and pain occurring in the stooping and squatting positions.

\section{Linked Angina}

It is an everyday observation that an anginal syndrome may be blended with pain or discomfort arising from non-cardiac sources. These combined disorders may offer much difficulty in assessment. Too often the patient (and his doctor) shy away from the unpleasant suspicion of angina by indicting other systems: thus anginal pain centred at the lower third of the sternum may be falsely attributed to gall-bladder disease, while cardiac pain radiating to the left shoulder and arm may be passed off as rheumatism. Clinical evasions of this sort are not only unsound but dangerous. The fact is that whenever serious consideration has to be given to such differential diagnosis there is a strong likelihood that both systems are involved. No satisfactory designation in English has yet been evolved for combined disorders of this kind. Froment (1955) has referred to them as "les angors coronariens intriqués." We do not believe that an English word or phrase with exactly the same connotation is to be found, and we shall refer to these complex syndromes as "linked angina "; this term emphasizes the connexion and at the same time names what is in most cases the major component.

No account need be given here of the well-known principles of referred pain which underlie linked angina. The fact that afferent paths from somatic regions (for example, shoulder and arm) or from visceral sources (for example, gall-bladder) enter the spinal cord at levels and by pathways closely corresponding to those of the afferent pathways for cardiac pain has been fully established. Other varieties of linked angina are those in which a coronary syndrome is intricately mixed with the symptoms of spinal arthritis, with hiatus hernia, with gastro-oesophageal reflux or disease, with gastric and duodenal ulcer, and with left mammary pain. The association of left submammary pain with true anginal symptoms is commonplace; no examples of this combination need be described.

In these linked anginas it is the rule for the coronary element to remain the major one. The paroxysmal pain is often unleashed by the non-coronary element of the combination, as when meals are interrupted by angina which may not be evoked by effort. Equally, the attack may be brought to an end by cessation of the provoking cause while nitrites may be ineffective. Froment (1955) has said that "every unquestionable angina that is provoked by certain specific and unusual conditions is most probably aggravated by an irritative non-coronary derangement of which the source determines the exact nature of the crises."

Among the most important linked anginas are those presenting a blend between coronary and gall-bladder or oesophageal symptoms. There is more experimental evidence pointing to the intimate connexion between these disorders than there is proof arising from clinical observation. Thus distension of the oesophagus and mechanical stimulation of the extrahepatic bile-ducts are both followed by reduction in the coronary blood flow. Hyperchlorhydria and duodenal lesions are frequent sources too often unsuspected. It is within the experience of most physicians that removal of a gallbladder containing stones may bring substantial relief from associated angina. One of us (K. S. S.) witnessed $2: 1 \mathrm{~A}-\mathrm{V}$ block develop during an attack of gall-bladder colic. This relationship in reverse has been emphasized by Levine (1958). In seven instances of Adams-Stokes disease he has had cholecystectomy performed without any death, and in some cases attacks of syncope have thereby been controlled. The following case reports illustrate some of the forms that linked angina may assume.

Case 9.-A man of 40 was a heavy smoker and suffered from indigestion. The latter suddenly became much worse and pain radiated to the left arm. A diagnosis of acute cholecystitis was made. When, eight days later, he was investigated a cardiac infarction was discovered. In 1957 and again in 1959 the dyspeptic symptoms were similar to those before the infarction: epigastric and lower sternal pressure, worsening during the night and unrelieved by glyceryl trinitrate. Discomfort might last for hours. A barium-meal examination at a local hospital in 1955 had shown no abnormality. There was never any pain on effort, although he was an active man. The only physical sign was slight duodenal tenderness. The cardiogram remained fixed, showing the old antero-septal transmural infarct. In 
1959 a barium-meal examination showed a small healed duodenal ulcer. This was evidently present in 1950 and modified the anginal pain of infarction. The nocturnal indigestion pain was due to myocardial ischaemia linked with the duodenal lesion.

Case 10.-In February, 1959, a housewife aged 52 complained of flatulence, pain on the left side of the chest, and palpitation. The symptoms appeared mostly after meals and woke her at night. They were relieved by belching and passing flatus. As both the cardiogram and the barium-meal examination were normal and as the patient's parents had died of early coronary disease, the symptoms were thought to be neurogenic and connected with anxiety. However, during the following months the patient developed angina of effort, a cardiographic effort test in December, 1959, being positive. In the next four months worsening angina of effort and frequent nocturnal angina, coupled with palpitation and abdominal distension, culminated in a syncopal attack. A few hours later a cardiogram showed subendocardial ischaemia, which in a few days led to a lateral infarct. Anticoagulant treatment was initiated and continued. $A$ barium-meal examination in May, 1960, disclosed a sliding hiatus hernia (Fig. 5) ; omission to employ tilting at the first examination had caused this to be overlooked. It cannot be doubted that, throughout, the symptoms have been triggered by the stimulus of the filled hernia and due to a link between this and the associated coronary heart disease.

Case 11.-A man of 52 began to have effort angina in 1953. The cardiogram showed ischaemic abnormalities brought out by the effort test. In 1955 he had.gall-bladder colic with sternal radiation of the pain. Although there was typical tenderness, $x$-ray examination of the gall-bladder and stomach gave negative results. Similar attacks recurred until 1958, when the patient experienced worsening nocturnal angina. In December of that year there were nightly attacks, and palpation over the gall-bladder revealed acute tenderness and at the same time provoked an extremely severe bout of angina which was relieved by glyceryl trinitrate. Ten minutes later the same palpation produced

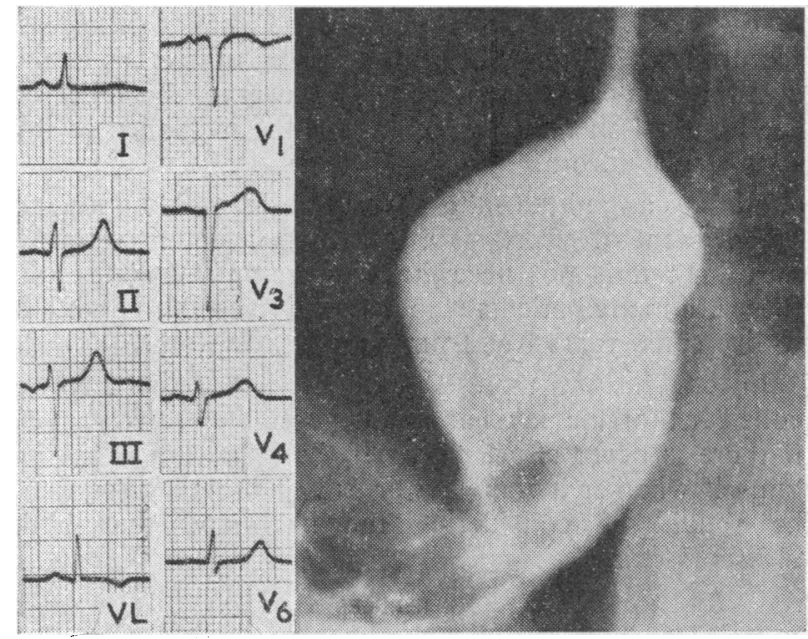

FIg. 5.-Case 10. Film of sliding hiatus hernia with contemporary electrocardiogram showing recovery from lateral infarct.

the same gall-bladder tenderness but no angina owing to the continuing effect of the vasodilator. In February, 1959, he had acute urinary retention and had to undergo an emergency prostatectomy. During the operation he developed cyanosis, tachycardia, and fall of blood-pressure. A few days later the cardiogram showed a recent anteroseptal infarct. He died a fortnight later in acute pulmonary oedema.

Case 12.-A man aged 52 developed angina of effort and a year later he had a cardiac infarction. In 1948 a second infarction was followed by continuing pain and disability in the use of the left shoulder resisting heat and physiotherapy.
Angina with or without effort persisted, becoming more and more inextricably linked with the shoulder symptoms, which now spread into the arm. In $1951 x$-ray examination showed cervical spondylitis involving C 5 and 6 (Fig. 6), and

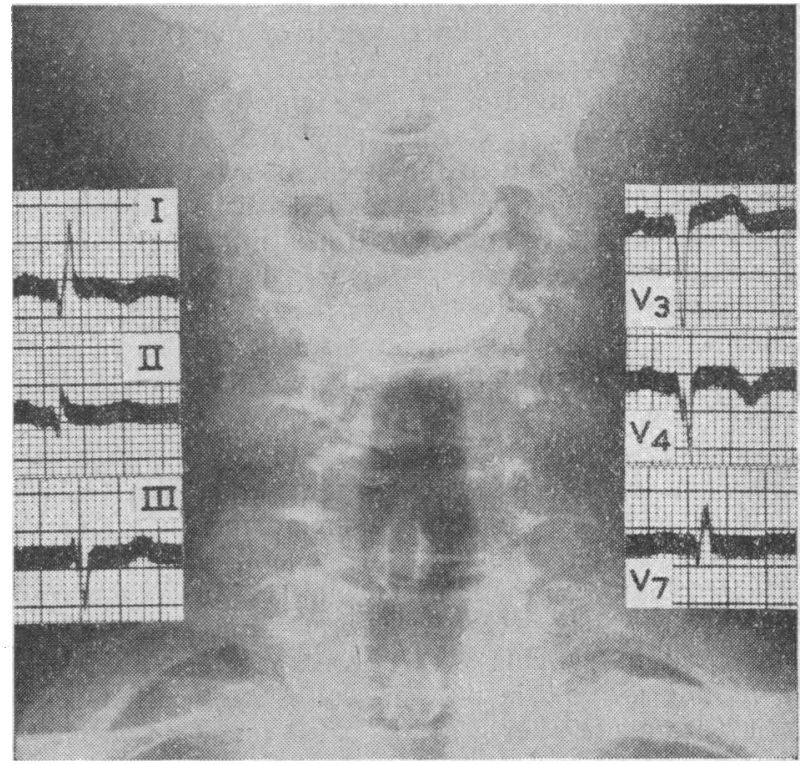

Fro. 6.-Case 12. Film of cervical spondylitis with contemporary electrocardiogram showing old anterior infarction.

neurological signs were discovered in the arm. This protracted and disabling illness, combining the ischaemic syndrome with that of cervical spondylitis, culminated in acute and fatal cardiac infarction in 1958.

\section{Discussion}

Consideration must now be given to the manner in which this clinical problem presents itself to the physician. The patient who is able to climb to his topfloor flat without discomfort may for years have had sporadic attacks of angina at night yet remained exceptionally active. This is different from angina at rest, as it has been described by clinicians, for it is neither pre-infarction angina nor the distressing status anginosus more often than not associated with disabling angina of effort (Papp and Shirley Smith, 1960). The housewife with known coronary disease may be fully active yet is unable to stoop because a painful lump spreading from the sternum to the jaw may make her choke.

The basic notion that all patients with angina have coronary artery disease and that therefore they can all be made to experience pain if subjected to adequate exertion (generally locomotion) has long held sway. It is the second part of this view that is here challenged. The full disproof is not easily made, since clinicians would not feel justified in subjecting a suspected coronary patient to prolonged or severe exertion; but the point is that certain people are having anginal seizures at rest and that concurrently they are leading full active lives with many unavoidable (and often sought) exertions, such as walking and climbing stairs, golfing and gardening, all with immunity from thoracic pain or distress.

Equally, the pain of angina can be evoked if one of the organs which has nervous connexion with the heart and which lies in its proximity has become diseased. This again presupposes a deficiency of the coronary circulation which may not necessarily be severe. The patient 
with known gastritis, duodenal ulcer, or hiatus hernia in whom "heartburn" spreads up the sternum and who is not relieved by the usual remedies ; the obese woman with gall-bladder disease in whom the attack radiates in an unusual way; the patient in the physiotherapy department whose frozen shoulder or spinal arthrosis is unsuccessfully treated, all of whom may be shown by the electrocardiograph to have coronary disease, are common examples. Cardiac pain is here closely interwoven with the pain of the diseased organ; there is no question of differential diagnosis, since both organs are responsible for the "mixed" pain of linked angina. The intensity and seriousness of the "linked" pain may depend on the balance between the degree of the extracardiac stimulus and the impairment of the coronary circulation. Such patients may be completely free of effort pain.

Knowledge of the interrelationship between the coronary system and the upper alimentary tract is derived from two main sources-clinical observation and animal experiments. A general review of the problem has been made by Giraud (1958). With regard to gastric reflexes, Gilbert (1942) showed that angina provoked in patients with coronary artery disease by breathing air low in oxygen would start earlier if the test were made after a meal than if made on an empiy stomach; this difference was not found when vagal influences were abolished by atropine. Hampton, Beckwith, and Wood (1959) have reviewed the evidence that the gall-bladder may initiate reflexes resulting in transient cardiographic changes, arrhythmias, and angina. It has been found by Ravdin, Royster, and Sanders (1942), who inserted T-tubes connected with a manometer into the bile-ducts of anginal patients, that raising the pressure in the ducts provoked angina. The correction of hiatus hernia by operation has been shown by De May, Cloetens, and Romainville (1959) to bring swift relief of coexistent angina, and such advantage must have been observed by numerous clinicians on many occasions.

Distension of viscera in the upper alimentary tract seems to be the most potent stimulus causing reflex effects upon the coronary circulation in animals. If the lower end of the gullet of an anaesthetized dog be distended, a $50 \%$ reduction in coronary flow may result (Daley, 1957) ; von Bergmann (1932) also demonstrated this relationship and found that the reflex decrease in coronary flow was specially notable if the distension was at the oesophageal hiatus; this effect was abolished by vagal section. Corresponding results are to be obtained by distension of the common bile-duct in the dog: a rapid fall in blood-pressure and reduction of the coronary flow follow mechanical stimulation of the extrahepatic bile-ducts or internal dilatation of the common bile-duct. Similarly, Cullen and Reese (1952), studying the coronary circulation in dogs by ${ }^{24} \mathrm{Na}$, showed that rapid distension of the common bile-duct lessened coronary flow if the vagi were intact but not if they had previously been cut in the neck.

To imagine that the mechanisms involved in the production of cardiac pain are complex is not difficult. Indeed, it would be astonishing if cardiac pain were simply a matter of discrepancy between myocardial demand and coronary supply. A physician who bases his conception of angina on analogies with irrigating systems in which conduits may become constricted or obstructed has a narrow view that must limit his understanding of these disorders. It has been shown that the nervous system exercises a direct control upon the heart-beat and the coronary system through a great complexity of higher centres, spinal centres and afferent and efferent pathways directly connected with the coronary arteries and linked with the sympathetic and somatic nerve supplies, and through nerve plexuses around the heart and great vessels. But even this is not the whole picture, since the coronary system and myocardium are under the control also of endocrine and nutritional and electrolytic influences and of baroceptors. The marvel is that this extremely complex mechanism is regulated and kept precisely in motion and out of consciousness in spite of the most variable and often unphysiological demands made upon it.

\section{Summary}

The realization after the first world war that pains below the left breast, independent of effort and often long-lasting, were not of cardiac origin placed, by contrast, too much emphasis on the direct relation of angina pectoris to effort. There are diverse forms of angina independent of effort, and a classification of these, based on current conceptions, is offered.

Attention is drawn to the group of patients who suffer paroxysms of angina at intervals over periods of months or years, by day or by night, but never in relation to effort. The difference between this episodic angina and angina of effort is discussed and the special problems connected with nocturnal angina are considered in the light of several examples.

Postural angina includes nocturnal angina and also angina of stooping. Some observations are made on the production of this latter disorder.

The association of coronary heart disease with noncardiac disorders that seem to trigger bouts of angina is discussed. This "linked angina" has the special feature that there is close interweaving of the ischaemic and the non-cardiac syndromes; however, the relief of the latter often remedies or lessens the incidence of the coronary syndrome.

Status anginosus-the severest form of angina independent of effort-is a term with a definite clinical meaning; it is not necessarily a pre-mortal state, and there are many patients who have survived this condition to live some years free from cardiac symptoms.

In conclusion, there should be less reluctance to consider thoracic pains at rest as possibly coronary, even if there is never effort pain. If the physician is asking himself whether it is angina or, say, gall-bladder disease, he should be considering the likelihood that both disorders exist.

\section{REFERENCES}

Bedford, D. E. (1958). Proc. 3rd Congrès mondial de Cardiologie Bruxelles, Abstract of Symposia, p. 314

von Bergmann, G. (1932). Dtsch. med. Wschr.. 58, 605

Cullen, M. L. and Reese, H. L. (1952). J. appl. Physiol., 5, 281. Daley, R. (1957). Brit. med. J., 2, 173 .

De May, D., Cioetens, W., and Romainville, P. (1959). Acta gastro-ent. beig., 22, 19.

Froment, R. (1955)' Brux.-méd., 35, 2429.

Gallavardin, L. (1925). Les Angines de Poitrine, p. 59. Masson, Paris.

Gilbert, N. C. (1942). Bull. N.Y. Acad. Med., 18, 83.

Giraud. G. (1958) Gastroenterologia (Basel), 90 ,

Hampton, A. G., Beckwith. J. R., and Wood, J. E., jun. (1959). Ann. intern. Mud., 50, 1135 .

Levine, S. A. (1958) "Clinical Heart Disease, 5th ed. Saunders, Philadelphia, London.

Papp, C., and Smith, K. S. (1960). Brit. Heart J., 22, 259.

Ravdin, I. S., Royster, H. P., and Sanders, G. B. (1942). Ann. Surg. 115, 1055

Reeves, T. J., and Harrison, T. R. (1956). J. chron. Dis., 4, 340. 